\title{
Stem Cell A Solution beyond the Pandemic Covid-19 the Uncertainty of being able to Move on
}

\author{
Mistrih $\mathrm{FG}^{1}$, Mistrih $\mathrm{NG}^{2}$ and Eghon $\mathrm{G}^{3 *}$ \\ ${ }^{1}$ General Director of Donka Avdaloff Clinic, Chile \\ ${ }^{2}$ Medical director Clinica Donka Avdaloff, Chile \\ ${ }^{3}$ President of Scientific Medical Association Chile ( ASOCIMED), University of Chile, Chile
}

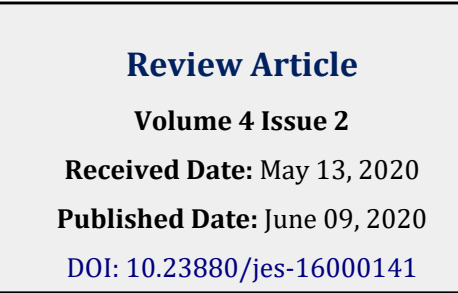

*Corresponding author: Eghon Guzman B, University of Chile, Chile; Email: eguzmanb@gmail.com

\section{Abstract}

In a market in Wuhan, China, apparently due to the ingestion of live bats, as part of the culture of the natives, an infection of the virus was generated, which until now has not been effectively treated, which is why we justify expanding our knowledge of a therapeutic alternative that appears, beyond being a preventive treatment, perhaps the solution to this pandemic, the effectiveness of IV mesenchymal cell transplantation, has not been described with conclusive results that allow scientists to propose it as the only treatment, but studies in multiple pathologies show a very favorable immune reaction, modulating inflammatory cascades in inflamed tissues. In addition, it clearly presents a regeneration of it (which is the same) that has powerfully caught the attention of doctors when treating pathologies, autoimmune, degenerative, and chronic pathologies, which to date have had no palliative treatments, such as SLE, post-infarction heart disease, chronic arthritis, Alzheimer's and among others, many laboratory studies were necessary, due to the urgent need of frustrated patients due to the few therapeutic possibilities that led to the use of mesenchymal cells for their treatment. The pneumonias caused by this new virus are of rapid progression and very lethal, with a treatment of little effectiveness and for this reason we have proposed the regulation of cytosines through the transplantation of mesenchymal cells, being these the main causes of complications due to their increase in the systemic reaction.

Keywords: Covid-19; Tissues; Receptor Bindings; Enzymes

Abbreviations: SARS: Severe Acute Respiratory Syndrome; MERS: Middle Eastern Respiratory Syndrome; ACE: Angiotensin-Converting Enzyme; WHO: World Health Organization; MSC: Mesenchymal/Stromal Stem Cell; TNF- $\alpha$ : Tumor Necrosis Factor $\alpha$; DAMP: Damage-Associated Molecular Patterns; ARDS: Acute Respiratory Distress Syndrome; SASP: Somatic Cell Senescence; SSCs: Senescent Somatic Cells.

\section{Introduction}

We have studied in different pathologies the use of stem cells to improve the quality of life of patients, but today we find a very different reality, beyond improving the functioning of organs and tissue regeneration as a preventive measure to devise a therapeutic alternative to a pandemic that compromises the lives of patients, without distinction of age, sex or ethnicity.

Towards the end of 2019 the world became aware of how vulnerable we could be if we did not take definitive measures; In the last two months of that year, a virus that until then was unknown [1-3] and that was quickly described as a type of coronavirus that was named by the WHO as covid-19, appeared in Wuhan, China. Caused by BETACORONAVIRUS called SARS-CoV-2, it was described as a relative of Severe Acute Respiratory Syndrome (SARS) and Middle Eastern Respiratory Syndrome (MERS), which affects 


\section{Journal of Embryology \& Stem Cell Research}

the lower respiratory system and presents as pneumonia [1], presenting Thus, a public health problem due to its rapid spread from person to person and its serious complications. In this review we will briefly explain the morphology of the virus and the reasons why it achieves its rapid transcription [2], causing the results we have seen on the rise, and which has unanimously forced all authorities to take drastic measures, such as quarantines.

We will describe a study of broncho alveolar fluid in hospitalized patients who had visited the Huanan seafood market in Wuhan (the place where the first cases of this pandemic came from), in these patients a genomic sequence of $2019-\mathrm{nCoV}$ is obtained, with a $99.9 \%$ similarity between them, also indicating that said phylogenetic examination identified it as the genus BETACORONAVIRUS derived from the bat found in eastern China, suggesting its origin as such $[3,4]$.

An enzyme called "angiotensin-converting enzyme 2" (ACE2) was found in the oral, nasal, nasopharyngeal mucosa, smooth muscles of the vascular endothelium, alveolar pulmonary epithelial cells, and enterocytes of the small intestine, which could be the functional receptor for SARS- CoV, although ACE2 mRNA is known to be present in almost all organs, which could explain its pathogenicity due to the fact that there is still no effective treatment, mortality remains high $[5,6]$. For this reason, we see the analysis of works that currently support the use of mesenchymal stem cells (stromal) for some pathologies that we will mention, pertinent and justified, focusing especially on patients hospitalized for pneumonia caused by the 2019-nCoV, such cell treatments Mesenchymal have not shown adverse effects which makes a very attractive treatment [7-14].

We will define some concepts in order to understand the immunological reaction that explains the reasons why the pro-inflammatory cascade caused by cytosines is modulated in patients with systemic damage in the context of infection by $2019-n C o V$, this article is also justified, with the review of experimental works in the first phase to treat pathologies such as SLE where it was shown that mesenchymal cells show immunosuppression in these cases, causing an improvement in symptoms and a decrease in the doses of the usual treatments. The anti-inflammatory effect caused by the release of the TSG- 6 protein due to pulmonary embolism of mesenchymal cells, which generate this reaction in patients who have suffered myocardial infarctions has been widely demonstrated, senescence also represents a problem when they appear degenerative diseases, immune system deficiency, so we have seen that the management of epigen through measures that include exercise, balanced diet, among others additionally, mesenchymal cell transplantation has proven to be a good treatment for give a better quality of life to patients in their old age, without having to suffer from the diseases of their age.

\section{Who Declares Global Emergency, before SARS- Cov2 Virus Pandemic}

In December 2019, an unprecedented outbreak of pneumonia of unknown etiology emerged in Wuhan City, Hubei Province of China. A new coronavirus was identified as the causative agent and was later named COVID-19 by the World Health Organization (WHO). Considered a relative of severe acute respiratory syndrome (SARS) and Middle East respiratory syndrome (MERS), COVID-19 is caused by a BETACORONAVIRUS called SARS-CoV-2 that affects the lower respiratory tract and manifests as pneumonia in humans. Despite rigorous global containment and quarantine efforts, the incidence of COVID-19 continues to rise, with more than 2 million laboratory-confirmed cases and more than 200,000 deaths worldwide. In response to this global outbreak, we summarize the current state of knowledge around COVID-19.

\section{Genomic Characterization and Epidemiology of the New Coronavirus 2019: Implications for Virus Origins and Receptor Binding}

A study was conducted in late December 2019, where patients who had viral pneumonia due to an unidentified microbial agent in Wuhan, China, and subsequently identified a new coronavirus as the causative pathogen, provisionally referred to as the new coronavirus 2019 ( Figures 1-3) (2019-nCoV) [2]. As of January 26, 2020, more than 2000 cases of 2019-nCoV infection have been confirmed, most of which involved people living or who visited Wuhan, and person-to-person transmission was confirmed.

The next generation of bronchoalveolar lavage fluid samples and cultured isolates from nine hospitalized patients, eight of whom visited the Huanan Seafood Market in Wuhan, were sequenced. Complete and partial genomic sequences of 2019-nCoV were obtained from these individuals. Viral contigs were connected using Sanger sequencing to obtain full-length genomes, with the terminal regions determined by rapid amplification of the cDNA ends. Phylogenetic analysis of these 2019-nCoV genomes and other coronaviruses was used to determine the evolutionary history of the virus and help infer its probable origin. Homology modeling was performed to explore the probable receptor binding properties of the virus.

The ten 2019-nCoV genome sequences obtained from the nine patients were extremely similar, exhibiting more than $99.9 \%$ sequence identity. In particular, 2019-nCoV was closely related (with $88 \%$ identity) to two bat-derived severe acute respiratory syndrome (SARS) coronaviruses, 


\section{Journal of Embryology \& Stem Cell Research}

bat-SL-CoVZC45 and bat-SL-CoVZXC21, collected in 2018 in Zhoushan, eastern China, but were more distant from SARSCoV (approximately 79\%) and MERS-CoV (approximately $50 \%)$. Phylogenetic analysis revealed that 2019-nCoV fell into the SARBECOVIRUS subgenus of the genus BETACORONAVIRUS, with a relatively long branch length to its closest relatives bat-SL-CoVZC45 and bat-SL-CoVZXC21, and genetically distinct from SARS-CoV. In particular, the homology model revealed that 2019-nCoV has a receptor binding domain structure similar to that of SARS-CoV, despite amino acid variation at some key residues.

The 2019-nCoV is divergent enough from the SARS$\mathrm{CoV}$ to be considered a new human BETACORONAVIRUS. Although our phylogenetic analysis suggests that bats may be the original host for this virus, an animal sold at the seafood market in Wuhan may represent an intermediate host that facilitates the appearance of the virus in humans.

Recently, a metallopeptidase called angiotensinconverting enzyme 2 (ACE2) has been identified as the functional receptor for SARS-CoV. Although ACE2 mRNA is known to be present in virtually all organs, its protein expression is largely unknown [6]. Since identifying the possible route of infection has important implications for understanding the pathogenesis and future treatment strategies for SARS, the location of the ACE2 protein was also identified in various human organs (oral and nasal mucosa, nasopharynx, lung, stomach, small intestine, colon, skin, lymph nodes, thymus, bone marrow, spleen, liver, kidney and brain). The most notable finding was the superficial expression of the ACE2 protein in pulmonary alveolar epithelial cells and enterocytes of the small intestine. Furthermore, ACE2 was present in arterial and venous endothelial cells and in arterial smooth muscle cells in all the organs studied.

In conclusion, ACE2 is abundantly present in humans in epithelia of the lung and small intestine, which could provide possible entry routes for SARS-CoV. This epithelial expression, together with the presence of ACE2 in the vascular endothelium, also provides a first step in understanding the pathogenesis of the main manifestations of SARS disease.
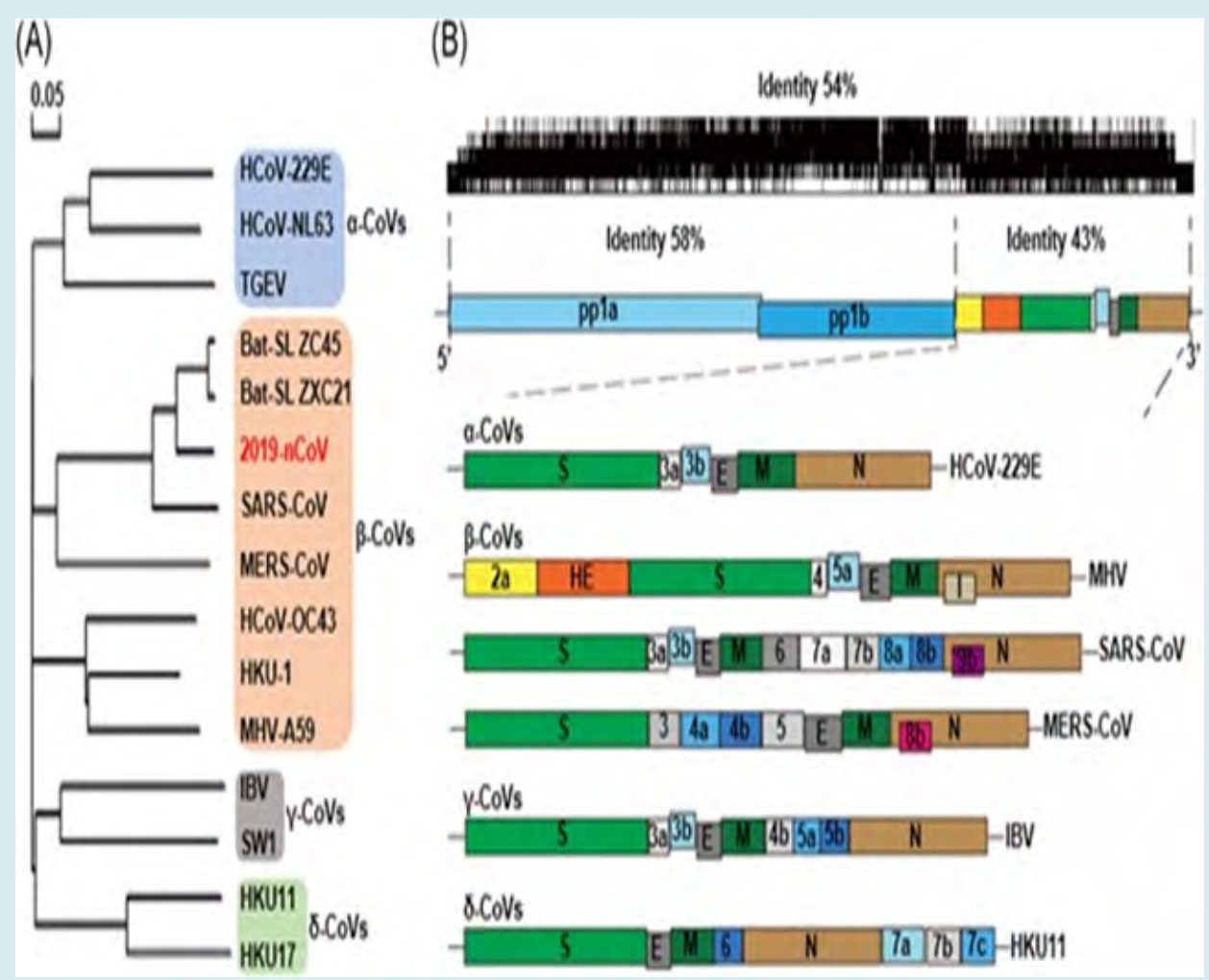

Figure 1: The genomic structure and phylogenetic tree of coronaviruses. A, The phylogenetic tree of representative CoVs, with the new coronavirus 2019-nCoV highlighted in red. B, The genome structure of four genera of coronaviruses. Pp1a and pp1b represent the two long polypeptides that are processed into 16 nonstructural proteins. S, E, M, and N indicate the four structural proteins spike, envelope, membrane, and nucleocapsid. 2019-nCoV, 2019 novel coronavirus; CoVs, coronavirus; HE, hemagglutinin-esterase. Viral names: HKU, coronaviruses identified by Hong Kong University; HCoV, human coronavirus; IBV, infectious bronchitis virus; MHV, murine hepatitis virus; TGEV, transmissible gastroenteritis virus. 


\section{Journal of Embryology \& Stem Cell Research}

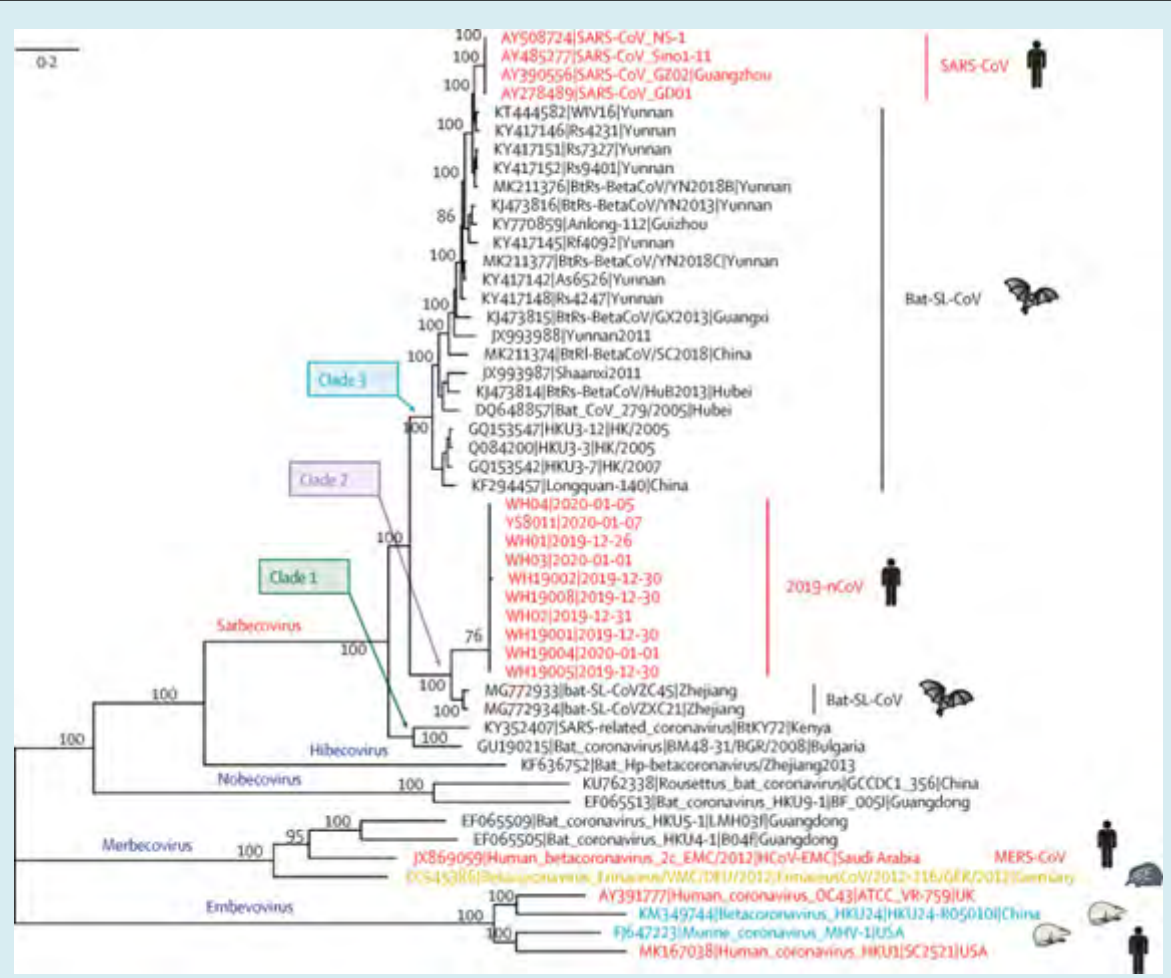

Figure 2: Phylogenetic analysis of full-length genomes of 2019-nCoV and representative viruses of the genus Betacoronavirus 2019-nCoV=2019 novel coronavirus. MERS-CoV=Middle East respiratory syndrome coronavirus. SARS-CoV=severe acute respiratory syndrome coronavirus.

\begin{tabular}{|c|c|c|c|c|c|c|c|c|c|c|c|c|c|c|c|}
\hline \multirow[t]{2}{*}{ A } & \multirow[t]{2}{*}{ Strain } & \multirow{2}{*}{$\begin{array}{c}\text { Complete } \\
\text { genome } \\
(\times)\end{array}$} & \multicolumn{13}{|c|}{ Gene region ( $(x)$} \\
\hline & & & lab & 12 & tb & 5 & 3 & E & $M$ & 7 & 8 & $10 \mathrm{~b}$ & $\mathrm{~N}$ & 13 & 14 \\
\hline $\begin{array}{l}\text { Nucleotide } \\
\text { sequences }\end{array}$ & $\begin{array}{l}\text { Bat-SL-CoVZC45 } \\
\text { Bat-SL-CoVzXC1 } \\
\text { SARS-CoVGZ02 }\end{array}$ & $\begin{array}{l}87.6 \\
87.5 \\
790 \\
\end{array}$ & $\begin{array}{l}88.9 \\
88.7 \\
795\end{array}$ & $\begin{array}{l}907 \\
90-3 \\
75-4\end{array}$ & $\begin{array}{l}86-0 \\
86-1 \\
86-3 \\
\end{array}$ & $\begin{array}{l}75.2 \\
747 \\
72.7\end{array}$ & $\begin{array}{l}87.8 \\
88.9 \\
756\end{array}$ & $\begin{array}{l}987 \\
987 \\
935 \\
\end{array}$ & $\begin{array}{l}93.4 \\
934 \\
85-1 \\
\end{array}$ & $\begin{array}{l}952 \\
952 \\
745 \\
\end{array}$ & $\begin{array}{l}88.8 \\
89.1 \\
82.1 \\
\end{array}$ & $\begin{array}{l}885 \\
885 \\
\end{array}$ & $\begin{array}{l}91.1 \\
91 \cdot 2 \\
88.1 \\
\end{array}$ & $\begin{array}{r}891 \\
895 \\
\end{array}$ & $\begin{array}{l}96-7 \\
96-7 \\
\end{array}$ \\
\hline $\begin{array}{l}\text { Amino acid } \\
\text { sequences }\end{array}$ & $\begin{array}{l}\text { Bat-SL-CoVZCAS } \\
\text { Bat-SL-CoVZXC21 } \\
\text { SARS-CoVGZO2 }\end{array}$ & - & $\begin{array}{l}95.6 \\
95.2 \\
86.2\end{array}$ & $\begin{array}{l}956 \\
951 \\
80.5\end{array}$ & $\begin{array}{l}958 \\
955 \\
956\end{array}$ & $\begin{array}{l}80.2 \\
796 \\
76-2\end{array}$ & $\begin{array}{l}90.9 \\
920 \\
73.1\end{array}$ & $\begin{array}{r}1000 \\
1000 \\
947\end{array}$ & $\begin{array}{l}986 \\
986 \\
901\end{array}$ & $\begin{array}{l}93.4 \\
934 \\
68.9\end{array}$ & $\begin{array}{l}87.6 \\
88.4 \\
85.2\end{array}$ & $\begin{array}{l}942 \\
942\end{array}$ & $\begin{array}{l}943 \\
943 \\
903\end{array}$ & $\begin{array}{l}732 \\
732\end{array}$ & $\begin{array}{l}92.9 \\
92.9\end{array}$ \\
\hline
\end{tabular}

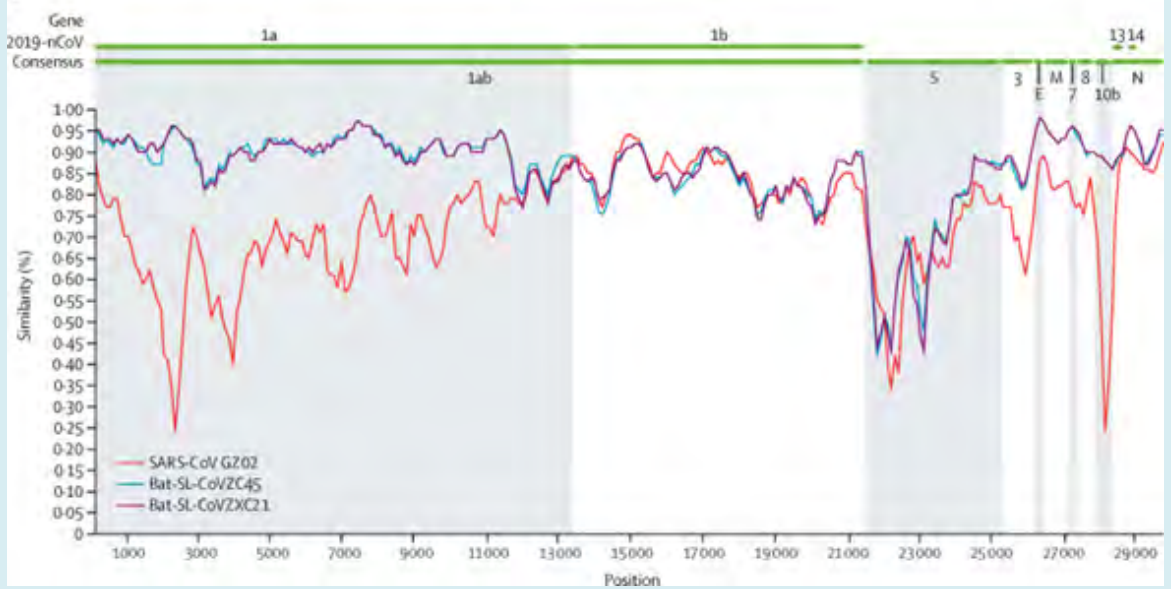

Figure 3: Sequence identity between the consensus of 2019-nCoV and representative betacoronavirus genomes (A) Sequence identities for 2019-nCoV compared with SARS-CoV GZ02 (accession number AY390556) and the bat SARS-like coronaviruses bat-SL-CoVZC45 (MG772933) and bat-SL-CoVZXC21 (MG772934). (B) Similarity between 2019-nCoV and related viruses. 2019-nCoV=2019 novel coronavirus. SARS-CoV=severe acute respiratory syndrome coronavirus. 


\section{Journal of Embryology \& Stem Cell Research}

\section{Mesenchymal Stem Cells/Stromal Cells (Mscs) and their Immune and Endocrine Function}

From the beginning it was clear that developing new mesenchymal/stromal stem cell (MSC) therapies was not a simple or easy task [6]. Among the first experiments was the administration of MSC from normal mice to transgenic mice that developed brittle bones because they expressed a mutated gene for type 1 collagen isolated from a patient with osteogenesis imperfecta. The results led to a clinical trial of MSC in patients with severe osteogenesis imperfecta. Subsequent work by a large number of scientists and physicians has established that, with minor exceptions, MSCs are not largely grafted or differentiated in vivo. Instead, cells produce beneficial effects in a large number of animal models and in some clinical trials by secreting paracrine factors and extracellular vesicles in a "hit and run" scenario. The field faces a number of challenges, but the results indicate that we are on the way to effective therapies for millions of patients suffering from devastating diseases.

Recent observations have shown that one of the functions of mesenchymal stem/stromal cells (MSCs) is to serve as gatekeepers against excessive inflammatory responses [8]. One mode of action for cells is that they are activated to express the interleukin receptor antagonist (IL) -1. A second mode of action is to create a negative feedback loop in which tumor necrosis factor $\alpha$ (TNF- $\alpha$ ) and other pro-inflammatory cytokines from resident macrophages activate MSCs to secrete the stimulated multifunctional anti-inflammatory protein TNF- $\alpha$ gene/Protein 6 (TSG-6). TSG- 6 then reduces nuclear factor $\kappa \mathrm{B}(\mathrm{NF}-\kappa \mathrm{B})$ signaling in resident macrophages, and thus modulates the cascade of pro-inflammatory cytokines. A third mode of action is to create a second negative feedback loop whereby lipopolysaccharide, TNF- $\alpha$, nitric oxide, and perhaps other damage-associated molecular patterns (DAMP) of injured tissues and macrophages activate MSCs to secrete prostaglandin E (2) (PGE). PGE [2] (Figure 1) converts macrophages into the phenotype that IL-10 secretes. There are also suggestions that MSCs may produce anti-inflammatory effects through additional modes of action, including activation to express the antireoreactive protein of the stannium-calcium-1 species.

\section{MSC for the Treatment of SARS}

There is no effective drug therapy for acute respiratory distress syndrome (ARDS), and mortality remains high. Preclinical studies support the efficacy of mesenchymal stem cells (stroma) in treating lung injury. A clinical study was carried out where the objective was to evaluate the safety of a single dose of allogeneic bone marrow-derived MSCs in patients with moderate to severe ARDS [14].
The Stem Cell Trial for Treatment of ARDS (START) was an open-label, phase 1, dose escalation, multicenter clinical trial. Patients were enrolled in the intensive care units of the University of California, San Francisco, CA, USA USA, Stanford University, Stanford, CA, USA, and Massachusetts General Hospital, Boston, MA, USA. Between July 8, 2013 and January 13,2014 . Patients were included if they had moderate to severe ARDS, as defined by the acute onset of the need for positive pressure ventilation by an endotracheal or tracheal tube, a PaO2: FiO2 less than $200 \mathrm{~mm} \mathrm{Hg}$ with at least 8 $\mathrm{cm} \mathrm{H} 2 \mathrm{O}$ positive end-expiratory airway pressure (PEEP) and bilateral infiltrates consistent with pulmonary edema on frontal chest radiograph. The first three patients were treated with low doses of MSC (1 million cells / kg expected body weight $[\mathrm{PBW}])$, the next three patients received intermediate doses of MSC ( 5 million cells / kg PBW), and all three Final patients received high doses of MSC (10 million cells / kg PBW). Primary outcomes included the incidence of pre-specified infusion-associated events and serious adverse events. The trial is registered with ClinicalTrials.gov, number NCT01775774.

No pre-specified infusion-associated events or treatment-related adverse events were reported in any of the nine patients. Subsequently, serious adverse events were observed in three patients during the weeks following the infusion: one patient died on day 9 of the study, one patient died on day 31 of the study, and one patient was found to have multiple strokes due to spleen, kidney embolism and the brain. They were undetermined by age, but are believed to have occurred prior to the MSC infusion based on the results of the MRI. None of these serious adverse events were thought to be related to SCD.

A single intravenous infusion of allogeneic bone marrowderived MSCs was well tolerated in nine patients with moderate to severe ARDS. Based on this phase 1 experience, we have proceeded to the phase 2 MSC test for moderate to severe ARDS with a primary focus on safety and secondary outcomes, including respiratory, systemic, and biological endpoints.

\section{Second Revision}

\section{MSC for Treatment of COVID-19 in the Context of Severe Pneumonia}

Preventing and reversing the cytokine storm may be the key to saving patients with severe COVID-19 pneumonia (...). Mesenchymal stem cells (MSCs) have been shown to possess powerful and comprehensive immunomodulatory function.

This study (...) aims to investigate whether MSC transplantation improves the outcome of 7 patients enrolled 


\section{Journal of Embryology \& Stem Cell Research}

with COVID-19 pneumonia at the Yuan Hospital in Beijing, China, from January 23, 2020 to February 16, 2020.

The clinical results, as well as the inflammatory and immune changes, the levels of function and the adverse effects of 7 included patients were evaluated for 14 days after the injection of MSC. MSCs could cure or significantly improve the functional outcomes of seven patients with no observed adverse effects. The lung function and symptoms of these seven patients improved significantly in 2 days after the MSC transplant. Among them, two common and one severe patient were recovered and discharged within 10 days after treatment. After treatment, peripheral lymphocytes increased, C-reactive protein decreased, and overactivated cytokine-secreting immune cells, CXCR3+CD4+T, CXCR3+CD8+T, and CXCR3+NK disappeared within 3-6 days. Furthermore, a group of $\mathrm{CD} 14+\mathrm{CD} 11 \mathrm{c}+\mathrm{CD} 11 \mathrm{bmid}$ regulating the DC cell population increased dramatically. Meanwhile, the TNF- $\alpha$ level decreased significantly, while IL-10 increased in the MSC treatment group compared to the placebo control group. Furthermore, the gene expression profile showed that the MSCs were ACE2 and TMPRSS2, indicating that the MSCs had no COVID-19 infection. Therefore, intravenous MSC transplantation was safe and effective for treatment in patients with COVID-19 pneumonia, especially for critically ill patients.

\section{The Impact of Immune Senescence on Viral Infection, Immunity and Immunogenicity of the Vaccine}

We know that the age group most vulnerable to the complications of covid-19arepeoplewith chronic diseases and especially the elderly, so we find it pertinent to study from an immunological perspective the aging of the immune system, which is characterized by the paradox of immunosenescence (insufficiency) and inflammation (overreaction), and which incorporate two sides of the same coin, resulting in an immune disorder [13]. Immunosenescence refers to disruption in the structural architecture of immune organs and dysfunction in immune responses, as a result of aging innate and adaptive immunity. Inflammation, described as a chronic, sterile, and systemic inflammatory condition associated with advancing age, is primarily attributed to the secretory phenotype associated with somatic cell senescence (SASP) and age-related autoimmune predisposition. However, the inability to reduce senescent somatic cells (SSCs), due to immunosenescence, exacerbates inflammation. Age-related deviations in the adaptive immune system, particularly altered T-cell function, stem from age-related thymic atrophy or involution, a hallmark of thymic aging. Recently, there have been important advances in understanding how agerelated thymic involution contributes to inflammation and immunosenescence at the cellular and molecular level, including genetic and epigenetic regulation, as well as the development of many potential rejuvenation strategies in Research progress to discover how thymic involution may be related to age and contributes to immunosenescence and inflammation, as well as their intersection. Both adaptive T-cell immunity mediates inflammation and plays a crucial role in the progression of age-related cardiovascular and neurological diseases, as well as cancer.

Multiple components of the innate and adaptive immune system undergo aging-related changes [11], such as alterations in the number of circulating monocytic and dendritic cells, reduced phagocytic activities of neutrophils, limited diversity in the B/T cell repertoire, exhaustion, or inflation of T cells, and chronic production of inflammatory cytokines known as inflammatory. The elderly are less likely to benefit from vaccines as preventive measures against infectious diseases due to the inability of the immune system to mount a successful defense. Therefore, aging is believed to decrease the efficacy and effectiveness of vaccines, suggesting a decrease associated with vaccination-induced immunogenicity aging.

In this review, we also discuss the changes associated with aging in innate and adaptive immunity and the impact of immunosenescence on viral infection and immunity. We further explore recent advances in strategies to improve immunogenicity of vaccines in the elderly. A better understanding of the molecular mechanisms underlying immunosenescence-related immune dysfunction will provide crucial insight into the development of effective vaccines and immunotherapies for older people.

\section{Conclusion}

Given that to date we do not have a definitive vaccine for covid-19, and that this pathology does not have an effective treatment, and it is also important to highlight that its complications are rapidly progressive and generate a health problem. We have proposed a therapeutic measure, which has been shown to have effective results in different inflammatory-type pathologies, as well as we have learned that the main complication of covid-19 is pneumonia due to the increased inflammatory reaction, and that we have also studied the effects adverse effects of mesenchymal cell transplantation, which appear to be null or very low, we found an important justification for the use of stem cells as an alternative treatment for hospitalized patients due to covid-19, being a solution for these complications, and having been studied in other pathologies, mesenchymal cell transplantation, should to start being considered therapeutic measure in the present and future, for degenerative diseases as well as inflammatory and different etiologies not only for covid-19. 


\section{Journal of Embryology \& Stem Cell Research}

It is imperative the use of stem cells to have a senescence far from degenerative, chronic inflammatory pathologies that generate a deterioration in the quality of life, so that reaching old age is not a cause of distancing from relatives due to this condition, without intentions of wanting to extend human life what could be considered an unethical issue.

This pandemic has definitely shown that we are as vulnerable a species as the others, that we inhabit the planet, but the use of stem cells could give us a great leap for human evolution.

\section{Conflicts of Interest}

The authors have no conflicts of interest

\section{References}

1. Sohrabi C, Alsafi Z, O'Neill N, Khan M, Kerwan A, et al. (2020) World Health Organization declares global emergency: A review of the 2019 novel coronavirus (COVID-19). Int J Surg 76: 71-76.

2. Chen Y, Liu Q, Guo D (2020) Emerging Coronaviruses: Genome Structure, Replication and Pathogenesis. 19(4): 418-423.

3. Zhou P, Yang XL, Wang XG, Hu B, Zhang L, et al. (2020) Cite this article A pneumonia outbreak associated with a new coronavirus of probable bat origin. Nature volume 579(7798): 270-273.

4. Lu R, Zhao X, Li J, Niu P, Yang B, et al. (2019) Genomic Characterisation and Epidemiology of 2019 Novel Coronavirus: Implications for Virus Origins and Receptor Binding. The Lancet 395(10224): 565-574.

5. Leng Z, Zhu R, Hou W, Feng Y, Yang Y, et al. (2020) Transplantation of ACE2- Mesenchymal Stem Cells Improves the Outcome of Patients with COVID-19
Pneumonia. Aging nad Disease 11(2): 216-228.

6. Hamming I, Timens W, Bulthuis MLC, Lely AT, Navis GJ, et al. (2004) Tissue distribution of ACE2 protein, the functional receptor for SARS coronavirus. A first step in understanding SARS pathogenesis 203(2): 631-637.

7. Prockop DJ, Oh JH (2012) Mesenchymal stem/stromal cells (MSCs): role as guardians of inflammation. Mol Ther 20(1): 14-20.

8. Prockop DJ (2017) The exciting prospects of new therapies with mesenchymal stromal cells. 19(1): 1-8.

9. Wilson JG, Liu KD, Zhuo H, Caballero L, McMillan M, et al. (2015) Mesenchymal Stem (Stromal) Cells for Treatment of ARDS: A Phase 1 Clinical Trial. 3(1): 24-32.

10. Shetty AK, Kodali M, Upadhya R, Madhu LN (2018) Emerging Anti-Aging Strategies - Scientific Basis and Efficacy. Aging Dis 9(6): 1165-1184.

11. Thomas R, Wang W, Su DM (2020) Contributions of agerelated thymic involvement to immunosenescence and inflammation. Immun Aging 17: 2.

12. Lee RH, Pulin AA, Seo MJ, Kota DJ, Ylostalo J, et al. (2009) Intravenous hMSCs Improve Myocardial Infarction in Mice because Cells Embolized in Lung Are Activated to Secrete the Anti-inflammatory Protein TSG-6. Cell Stem Cell 5(1): 54-63.

13. Oh SJ, Lee JK, Shin OS (2019) Aging and the Immune System: the Impact of Immunosenescence on Viral Infection, Immunity and Vaccine Immunogenicity. Immune Netw 19(6): e37.

14. Wilson JG, Liu KD, Zhuo H, Caballero L, McMillan M, et al. (2015) Mesenchymal Stem (Stromal) Cells for Treatment of ARDS: A Phase 1 Clinical Trial. Lancet Respir Med 3(1): 24-32. 